\title{
Gut Formation after Transection of the Midgut Loop in the Chick Embryo
}

\author{
By
Hirotsugu TERAKURA, Kazuya YOSHINAGA*, Yoshihisa SERA and Toyoaki FUJIMOTO*

\author{
Second Department of Surgery and Department of Anatomy*, \\ Kumamoto University Medical School, Kumamoto 860, JAPAN \\ - Received for Publication, February 16, 1990-
}

\begin{abstract}
Key words: Midgut loop, Rotation, Fetal surgery, Chick embryo, Out-of-the-shell incubation
Summary: The primitive gut in vertebrates can be divided into the foregut, midgut, and hindgut. The midgut forms the midgut loop or the intestinal loop, which rotates as it develops the small and large intestines. We examined the effects of transection of the midgut loop on the subsequent development of the intestine in chick embryos by their out-of-the-shell incubation. The development of the embryo and the intestines out of the shell was nearly identical to that usual in-the-shell incubation. The rotation of the midgut loop began in stage 27 (5 days of incubation) and was completed in stage 36 (10 days of incubation) after counterclockwise rotation through $180^{\circ}$. In experimental groups, the midgut loop was transected immediately before or in the early stages of rotation (stages 27-33) proximally or distally to the apex of the loop. Transecting of the midgut loop caused little effects on its subsequent rotation regardless of the time or the site of transection although the secondary loop formation of intestine was poor in some cases. The stumps of the transected intestine were repaired and closed. After closure of the stumps, the proximal segment of the intestine was dilated in some cases. The secondary loop formation, or convolution of the intestine didn't occur in some dilated cases.
\end{abstract}

Congenital intestinal atresia or stenosis and morphological or positional abnormalities of the intestines based on malrotation of the midgut are frequent anomalies of the intestines in pediatric surgery. Experimental operations of embryos in various animals have been performed for investigating of the causes of these anomalies. Louw et al. (1955) demonstrated in the dog that congenital atresia of the small intestine was caused by mesenterial circulatory impairment. In other species of mammals, many embryonal experimental operations have been carried out for analyzing the mechanisms of intestinal atresia (lamb, Pickard et al., 1981, Doolin et al., 1987; rabbit, Wesson et al., 1984), gastroschisis (rabbit, Aoki et al., 1980, 1981), diaphragmatic hernia (sheep, Soper et al., 1984), and of obstructive jaundice (rabbit, Kawarasaki, et al., 1981) with some success.

Although the derivatives of the foregut in birds are somewhat different from those in mammals and the birds lack the umbilicus, the development of the midgut and its more distal part is nearly identical between birds and mammals. We used chicks as experimental animals because their embryos can be developed in culture out- of-the shell, so they are advantageous for visual evaluation of the process of the intestinal formation. In fact the chick embryos have been used for investigating the causes of congenital anomalies (intestinal atresia, Tibboel et al., 1981, 1982, Molenaar et al., 1982; gastroschisis, Kluck et al., 1983, Tibboel et al., 1985, 1986; Hirschsprung's disease, Meijers et al., 1989).

In amniotes the development of the intestine begins with formation of the midgut loop in the embryonic period and completes with the subsequent rotation and elongation of the loop. However, the process of the intestinal formation has been relatively poorly documented in the chick embryo, and comparisons between in-the-shell and out-of-the-shell developments have scarcely been made (Perry, 1988). Therefore, we planned to study the formation of the intestines in the chick embryo, especially rotation of the midgut loop and its subsequent development by in- and out-of-theshell incubation and, then, examined the effects of transection of the midgut loop, on the subsequent rotation and development along with repair or atresia of the stumps of the transected ends. 


\section{Materials and Methods}

The materials used for the present study were White Leghorn chick embryos.

(1) The process of intestinal formation was studied in normal embryos incubated in the shell at $38^{\circ} \mathrm{C}$ by the routine manner. A total of 115 embryos, namely 5 embryos of each stage from stage 24 to 46 (by Hamburger \& Hamilton, 1951), from 4 days to 21 days of incubation, were evaluated.

(2) Fifty embryos (as described below) were incubated out-of-the-shell, and their development was compared with that of embryos incubated in the shell. Embryos in stage 15-17, 2.5 days of in-the-shell incubation were aseptically removed from the shell; they were transferred to Petri dishes, placed in an incubator at $38 \pm 0.2^{\circ} \mathrm{C}$ and $100 \%$ humidity, continued to culture and observed at the various developmental stages until stage 40 . Transection of the midgut loop was carried out under these conditions as described below.

(3) Transection of the midgut loop: The egg was incubated without turning around to allow the embryo proper to stay above the yolk. No culture medium was placed in the Petri dishes to which the embryos were transferred after their removal from the shells. The midgut loop was transected in stage 27 (5 days of incubation) immediately before rotation of the loop and in stage 30-33 (7-8 days of incubation) during rotation under stereoscopic microscopy using forceps. First, the chorio-allantoic membrane was incised with minimum damage to the vessels. Even when there was mild hemorrhage, hemostasis was observed quickly. This procedure exposed the amnion covering the embryo. Incision of the amnion of the umbilical stalk revealed the midgut loop, and the omphalo-mesenteric artery, running toward the apex of the loop, was visible. The midgut loop was cut with a pair of forceps while holding it with another pair. The site of transection was slightly proximal or distal to the apex of the loop, i.e. slightly proximal or distal to the intestinal attachment of the omphalo-mesenteric artery (Fig. 1). After transection of the loop, the stumps were separated as much as possible. The chorio-allantoic membrane and the amnion were kept intact, and incubation of the embryos was continued after operation. The operation was performed in 127 embryos, and, excluding 40 that died within 24 hours, the remaining 87 were available for evaluation. The embryos could be incubated until stage 40 at the maximum. The embryos were treated with Bouin's fixative or fixed in $10 \%$ formalin solution and grossly observed by dissection under the stereoscopic microscope. Histological examinations of the intestine were also performed. Sections were cut at $5-\mu \mathrm{m}$ in thickness, double stained with hematoxylin and eosin and they were examined light-microscopically.

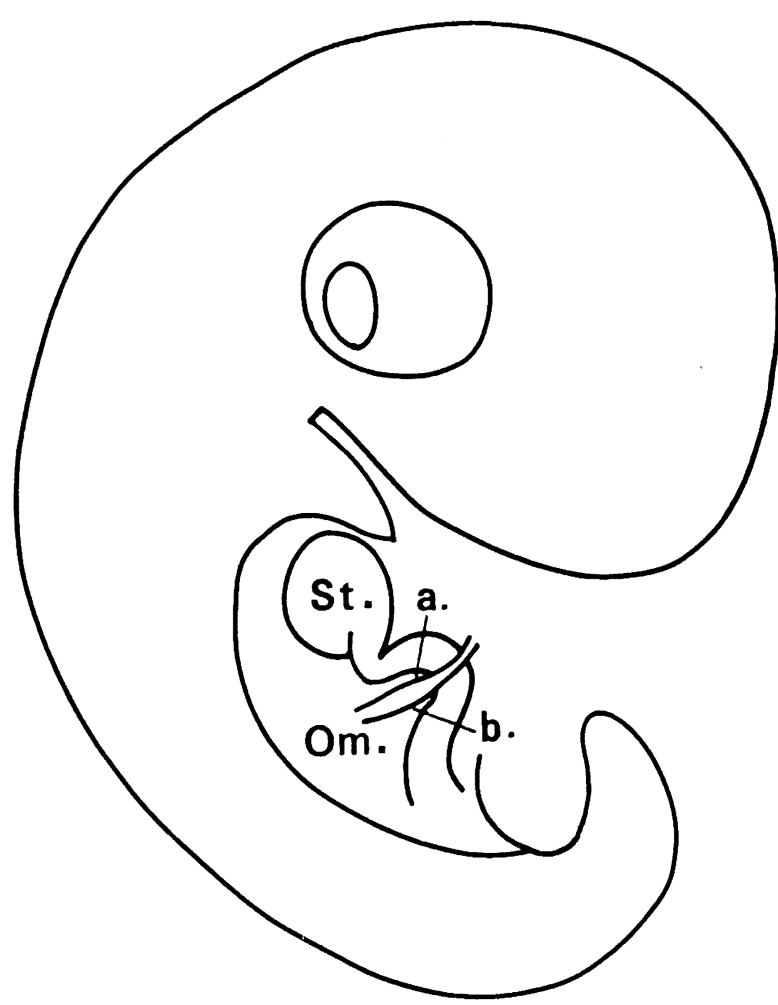

Fig. 1. Transection of the midgut loop was made slightly proximal (a.) or distal (b.) site to the apex of the loop, at which the omphalo-mesenteric artery (Om.) attaches St.; Stomach

\section{Results}

(1) Process of intestinal development in normal in-theshell embryos

The L-shaped midgut, the midgut loop formation appeared on stage 27 (5 days of incubation). At this stage the omphalo-mesenteric artery, running toward the apex of the loop in the mesentery, was clearly visible (Fig. 2). The portion of the midgut loop cephalic to its attachment to the omphalo-mesenteric artery is hereafter referred to as the proximal limb and that caudal to the attachment as the distal limb. Rotation of the loop began in stage 28 (5.5 days of incubation); only the proximal limb slightly rotated counterclockwise around the omphalo-mesenteric artery (Fig. 3). The intestine became elongated thereafter, and in stage 31 (7 days of incubation), both the proximal and distal limbs rotated counterclockwise through $90^{\circ}$ around the omphalo-mesenteric artery, and both the proximal and distal limbs became horizontal (Fig. 4). In this process of horizontalization, the distal limb, of which the initial rotation was delayed, moved more rapidly than that in the proximal limb, having rotated already through $90^{\circ}$ in stage 30 ( 6.5 days of incubation). Both the proximal and distal limbs rotated symmetrically counterclockwise thereafter, and the proximal limb finally 
shifted below the distal limb in stage 36 (10 days of incubation) as a result of $180^{\circ}$ rotation. Thus the rotation of the loop was completed at this stage (Fig. 5). Subsequently, the intestine was elongated gradually, and both limbs formed secondary intestinal loops by convoluting 5-6 times. This process terminated in embryos after stage 40 (14 days of incubation) (Fig. 6). No further convolution was observed, and the intestine grew in length and width thereafter.

Histological aspects: At stage 27, the muscle layer in the wall of the midgut loop was not observed, although the mucosa, submucosa and serosa were distinguished (Fig. 7). At stage 31, both the annular and longitudinal muscle fibers became appeared, thus forming three layered structure (mucosa, muscle layers and serosa) as in the adult gut (Fig. 8). At stage 33, the luminal surface of mucosa was slightly undulated, roughly folded and the formation of villi had begun (Fig. 9). At stage 35, the villus formation had progressed, and the inner circular and outer longitudinal muscle layers had well developed (Fig. 10). At stage 39, well-developed villi were observed, and the muscle layers became more thickened like the structure of adults form (Fig. 11). The histological differentiation of the intestine started in the cephalic portion of the midgut and progressed caudally.

(2) Development of the embryo and its midgut by outof-the-shell incubation; Comparisons with in-the-shell incubation

The development of embryos incubated out-of-theshell was identical to that of embryos incubated in-theshell in terms of the relationship between the incubation time and the degree of development (developmental stage by Hamburger and Hamilton, 1951) until 13 days of incubation. However, the embryonic development showed little progression thereafter and was arrested at about stage 40 (Table 1). The development of the intestines of embryos incubated out of the shell was similar to that of embryos incubated in the shell; midgut rotation and the subsequent secondary intestinal loop formation were performed corresponding to the normal stages of the embryonic development.

(3) Intestinal development after transection of the midgut loop

Eighty-seven of the 127 embryos transected the midgut loop were survived and developed to a maximum of stage 40. Embryos that were viable more than 24 hours after operation were examined. Among them, the embryos survived until stage 36 or further, when rotation of the midgut loop was completed, and allowed observation of the entire process of midgut rotation were as follows: 9 of 27 embryos operated at stage 27, 17 of 30 embryos operated at stage 31,16 of 31 embryos operated at stage 32 , and 23 of 39 embryos operated at stage 33 . In the above embryos, the incisions in the chorio-allantoic membrane and the amnion were closed
Table 1. Comparison of embryonic development between in-theshell and out-of-the-shell incubation

\begin{tabular}{lcc}
\hline & \multicolumn{2}{c}{ Stages* } \\
\cline { 2 - 3 } Days of Incubation & in-the-shell & out-of-the-shell \\
\hline 5.0 & 27 & 27 \\
5.5 & 28 & 28 \\
6.0 & 29 & 29 \\
6.5 & 30 & 30 \\
7.0 & 31 & 31 \\
7.5 & 32 & 32 \\
$7.5-8.0$ & 33 & 33 \\
8.0 & 34 & 34 \\
$8.0-9.0$ & 35 & 35 \\
10.0 & 36 & 36 \\
11.0 & 37 & 37 \\
12.0 & 38 & 38 \\
13.0 & 39 & 39 \\
14.0 & 40 & 39 \\
15.0 & 41 & 40 \\
16.0 & 42 & $40^{* *}$ \\
17.0 & 43 & $40^{* *}$ \\
18.0 & 44 & $40^{* *}$ \\
$19.0-20.0$ & 45 & $40^{* *}$ \\
$20.0-21.0$ & 46 & $40^{* *}$ \\
\hline
\end{tabular}

* Hamburger \& Hamilton, 1951.

** survived but developmental stage was not advanced

spontaneously.

(1) Closure of stumps

As mentioned above, the midgut was transected slightly proximal or distal to the apex of the loop at stage 27 immediately before the beginning of rotation of the loop and at stages 31,32 , and 33 during rotation. The stumps were closed regardless of the time or the site of the operation as described below. (Table 2).

1. Closure of stumps: Both proximal and distal transected ends were closed in 50 (about $40 \%$ ) of the 127 embryos after resection of the midgut loop at stage 28 or later. In most embryos, the two stumps were completely detached, and each stump was closed (this type of closure of each separated stamp is classified as type III in terms of pediatric surgery, Louw, I.H., 1966), but in part of the embryos, the two stumps were in contact, and each was closed by a membranous structure (type I, as described above). This is due to tissue repair in the stump in either case.

2. Open stumps: Both proximal and distal transected ends remained open without closing in 25 embryos (about 20\%). Despite transection, growth of the intestine was observed as in normal in 12 embryos. In these embryos, separation of stumps after transection is considered to have been insufficient so that the stumps came into contact with each other, fused, and became continuous. 
Table 2. Effects of transection of the midgut loop of chick embryo just before (Stage 27) or on the way of its rotation

\begin{tabular}{ccccccc}
\hline \multirow{2}{*}{$\begin{array}{l}\text { Operated } \\
\text { stage }\end{array}$} & \multicolumn{2}{c}{ number of } & & \multicolumn{2}{c}{ number of embryos with } \\
\cline { 2 - 3 } \cline { 5 - 6 } & $\begin{array}{l}\text { operated } \\
\text { embryos }\end{array}$ & $\begin{array}{l}\text { death } \\
\text { within } 24 \mathrm{hr} .\end{array}$ & $\begin{array}{l}\text { atresia of } \\
\text { intestine } \\
<\text { dilatation }\end{array}$ & no atresia & $\begin{array}{l}\text { normally } \\
\text { developed }\end{array}$ \\
\hline 27 & 27 & 8 & $8<2>$ & 6 & 6 \\
31 & 30 & 8 & & $11<4>$ & 8 & 3 \\
32 & 31 & 10 & & $14<4>$ & 5 & 2 \\
33 & 39 & 14 & & $17<10>$ & 6 & 2 \\
total & 127 & 40 & & $50<20>$ & 25 & 12 \\
$\%$ & 100 & 31.5 & $39.4<40.0>$ & 19.7 & 9.4 \\
\hline
\end{tabular}

3. Dilatation of stumps: The intestine was dilated proximally to the transection site in 20 of the 50 embryos in which the stumps were closed as in 1 (Figs. $13 \& 14)$. The degree of dilatation was greater as the time after transection was longer. In two embryos autopsied 6 days and 8 days after operation, dilatated portion of the intestine was ruptured. Retention of meconium was observed in most of the dilated intestines.

4. Comparisons between transections proximal and distal to the midgut loop apex (Table 3): Intestinal atresia was noted in 37 of 66 embryos in which the midgut loop was transected proximally to the loop apex before or in the course of rotation, and the intestine was dilated in $20(54.1 \%)$ of these embryos. Intestinal atresia was observed in 13 of 31 embryos in which the midgut loop was transected distally to the loop apex before or at the beginning of rotation, but none of these embryos showed dilatation of the intestine. Closure of the stumps was accompanied by dilatation only in those that underwent proximal loop transection.

5. Closure of stumps and histological repair: In 25 embryos, the stumps were examined grossly and histologically 1, 2, 4, 8, 16, 24, 27, 48, and 72 hours after transection. Closure of the stumps was complete already 8 hours after operation in some embryos and within 48 hours at the maximum.

Closure of stumps was generally associated with the histological repair. Histological repair of the stumps occurred gradually, and with closure of the stumps, the three-layered structure of the mucosa, muscle layer, and serosa was formed simultaneously with the normal histogenesis in intact portions of the intestine. In dilated portions, this three-layered structure was also observed, but the intestinal wall was generally thinned (Fig. 12). (2) Rotation of the midgut loop and subsequent secondary loop formation

The timing and extent of rotation of the proximal and distal limbs of the midgut loop were identical to those observed in normal embryos whether the loop was transected at stage 27 (immediately before the beginning of rotation), at stage 31 (early stage of rotation), or stage 33 (late stage of rotation), or whether it was transected proximally or distally to the apex of the loop. On the other hand, in the cases that showed dilated stumps, the subsequent formation of the secondary loop

Table 3. Relationship of transected position of the midgut loop to its subsequent atresia

\begin{tabular}{|c|c|c|c|c|c|c|c|}
\hline \multicolumn{4}{|c|}{ proximal limb of the loop was transected } & \multicolumn{4}{|c|}{ distal limb of the loop was transected } \\
\hline \multirow{2}{*}{$\begin{array}{l}\text { Operated } \\
\text { stage }\end{array}$} & \multirow{2}{*}{$\begin{array}{l}\text { number of } \\
\text { operated } \\
\text { embryos }\end{array}$} & \multicolumn{2}{|c|}{ number of embryos with } & \multirow{2}{*}{$\begin{array}{l}\text { Operated } \\
\text { stage }\end{array}$} & \multirow{2}{*}{$\begin{array}{l}\text { number of } \\
\text { operated } \\
\text { embryos }\end{array}$} & \multicolumn{2}{|c|}{ number of embryos with } \\
\hline & & $\begin{array}{c}\text { atresia of } \\
\text { intestine } \\
<\text { dilatation }>\end{array}$ & no atresia & & & $\begin{array}{c}\text { atresia of } \\
\text { intestine } \\
<\text { dilatation }>\end{array}$ & no atresia \\
\hline 27 & 21 & $6<2>$ & 3 & 27 & 6 & $2<0>$ & 3 \\
\hline 31 & 12 & $9<4>$ & 2 & 31 & 7 & $2<0>$ & 3 \\
\hline 32 & 10 & $6<4>$ & 1 & 32 & 13 & $8<0>$ & 2 \\
\hline 33 & 23 & $16<10>$ & 3 & 33 & 5 & $1<0>$ & 2 \\
\hline total & 66 & $37<20>$ & 9 & & 31 & $13<0>$ & 10 \\
\hline$\%$ & 100 & $56.1<54.1\rangle$ & 13.6 & & 100 & $41.9<0>$ & 32.2 \\
\hline
\end{tabular}


did not occur, and the intestine developed without convolution (Figs. $13 \& 14$ ), but it progressed as in normal embryos when dilatation was absent or poor (Fig. 15). Therefore, rotation of the midgut loop and the subsequent secondary loop formation are not considered to have been impaired whether the midgut loop was transected before or during rotation as long as there was no dilatation of stumps. There were no marked differences in the histological profile of the transected midgut as compared with the normal embryos during secondary loop formation except in the dilated portion.

\section{Discussion}

The primitive alimentary canal, or the entodermal digestive tract in vertebrates, can be divided into the foregut, midgut, and hindgut. The midgut forms the so-called midgut loop, which develops into the small and large intestines as it is rotated, elongated and convoluted. There has been little sequential documentation of the process of gut formation in the chick embryo. Our study showed that rotation of the midgut loop begins at stage 28 (5.5 days of incubation) of the embryonal development, and is completed by stage 36 (10 days of incubation) after the loop was rotated through $180^{\circ}$ counterclockwise. The adult stage is attained after 5-6 secondary convolutions due to subsequent growth of the intestine in length and width. In humans, the midgut loop first prolapses into the umbilical celom, rotates $180^{\circ}$ counterclockwise, and then return into the abdominal cavity, where it rotates further by $90^{\circ}$ to a total of $270^{\circ}$ (Snyder, 1954). In the mean time, the intestines with and without mesentery develop, and the position of each intestinal segment is determined. In chicks, the extent of rotation is smaller, and, thus, the relationship between the mesentery and the intestine is not as complex as in humans. The chick intestine has the mesentery nearly in its entire length and is attached to the posterior peritoneal wall only in the colon (distal part of the attachment of appendix) (Kato, 1974). As for how this intestinal rotation occurs, i.e. the mechanism of rotation, is still ambiguous. In this study, we attempted first to clarify the process of midgut loop rotation and then to examine the effects of transection of the early midgut loop on the subsequent development or rotation of the intestines.

Most studies in the chick embryo to date have been usually carried out by in-the-shell procedures. In the present study, by out-of-the-shell incubation, embryos could be developed to stage 40 , and the process of intestinal development could be observed under the stereoscopic microscope along with the development of other organs. Thus, the out-of-the-shell incubation was very useful for a kind of study as we performed.

With regard to the intestinal rotation or convolution,
Mall et al. (1898) described that the small and the large intestines increase their length rapidly in development, but the space in which they should occupy within the body cavity remains the same. So, the intestine would make room for itself within the body cavity by rotating, probably due to mechanical causes. This concept will be applicable to initial loop rotation and secondary loop (convolution) formation in the chick.

Concerning the effects of transection of the midgut loop, rotation of the midgut loop proceeded after transection as in in-the-shell embryos or control outof-the-shell non transected embryos whether transection was performed before (stage 27) or during (stage $31-35$ ) rotation or whether the site of transection was proximal or distal to the apex of the loop. It is noticeable when transection was performed at stage 27 (before the beginning of rotation), the subsequent rotation took place as in the normal embryo. This suggests that the intestinal rotation is dependent on other factors than the loop itself, possibly it involves the movement of the mesentery as one of the factors. Moreover, rotation of the loop occurred as in usual manner even in cases with closure and dilatation of the stumps after intestinal transection. Therefore, it is considered that a complex mechanism is involved in intestinal rotation. This remains unsolved in the future problem for us.

In this study, the stumps after transection of the midgut loop were repaired in a short period, resulting in their closure. Tibboel et al. (1981) approached the mechanism of congenital stenosis or atresia of the human small intestine by using chick embryo as materials. They tried to perforate the intestine in 9-dayold embryo of in-the-shell incubation and observed to occur stenosis or atresia at the site of perforation after 48 hours. In our study, repair and closure of the stumps occurred within 8 hours of transection. Although it is difficult to compare at the same plane between the two experiments, this time lag may be due to the operation time; we treated so early stage as 5 to 7 days of incubation that histological repair of the stumps occurred according to the normal intestinal histogenesis.

As mentioned in the results section, when the midgut loop was transected in a proximal portion to its tip, dilatation of the intestine proximal to the closed stump was observed. This dilatation is considered to have occurred due to retention of the intestinal contents such as meconium with the developmental progress.

Viewing from congenital anomalies, such as stenosis or atresia of the intestine in man, for example, it is unknown that a phenomenon as transection of the midgut loop as a cause of these anomalies would occur spontaneously during the embryonic development. However, the present study has provided some idea for future experimental approaches to intestinal anomalies. 


\section{References}

1) Aoki, Y., T. Ohsio and N. Komi: An experimental study on gastroschisis using fetal surgery. J. Pediatr. Surg., 15: 252-256, 1980.

2) Aoki, Y., T. Ohsio and N. Komi: An experimental study on gastroschisis. Jap. J. Pediat. Surg., 13: 1315-1321, 1981 (in Japanese).

3) Doolin, E.J., H.S. Ormsbee and J.L. Hill: Motility abnormality in intestinal atresia. J. Pediatr. Surg., 22: 320-324, 1987.

4) Hamburger, V. and H.L. Hamilton: A series of normal stages in the development of the chick embryo. J. Morph., 88: 49-67, 1951.

5) Kato, Y. ed.: Intestine and cloaca in the chicken. In "Comparative Anatomy of the Domestic Animals", Vol. 1, 248-249, Kenseidou, Tokyo, 1974 (in Japanese).

6) Kawarasaki, H., T. Hori, T. Kamiyama, S. Harada, K. Yoshimura and Saypehth: Fetal surgery of the rabbit: Basic investigation about the etiology of infantile obstructive jaundice. Jap. J. Pediat. Surg., 13: 1331-1339, 1981 (in Japanese).

7) Klück, P., D. Tibboel, A.W.M. van der Kamp and J.C. Molenaar: The effect of fetal urine on the development of the bowel in gastroschisis. J. Pediatr. Surg., 18: 47-50, 1983.

8) Louw, J.H. and C.N. Barnard: Congenital intestinal atresia. Observations on its origin. Lancet 2: 1065-1067, 1955.

9) Louw, J.H.: Jejunoileal atresia and stenosis. J. Pediatr. Surg., 1: 8-23, 1966.

10) Mall, F.P.: Development of the human intestine and its position in the adult. Bull. Johns Hopkins Hops., 9: 197-208, 1898.

11) Molenaar, J.C. and D. Tibboel: The pathogenesis of atresias of the small bowel and colon. South Africa J. Surg., 20: 87-95, 1982.

12) Meijers, J.H.C., D. Tibboel, A.W.N. van der Kamp, I.C.C.M van Haperen-Heuts and J.C. Molenaar: A model for aganglionosis in the chicken embryo. J. Pediatr. Surge, 24: 557-561, 1989.

13) Perry, M.M.: A complete culture system for the chick embryo. Nature, 331: 70-72, 1988.

14) Pickard, L.R., S. Santoro, R.G. Wyllie and J.A. Haller, Jr.: Histochemical studies of experimental fetal intestinal obstruction. J. Pediatr. Surg., 16: 256-260, 1981.

15) Snyder, W.H. Jr. and L. Chaffin: Embryology and pathology of the intestinal tract: Presentation of 40 cases of malrotation. Annals of Surgery, 140: 368-380, 1954.

16) Soper, R.T., K.C. Pringle and J.C. Scofield: Creation and repair of diapfragmatic hernia in the fetal lamb: Techniques and survival. J. Pediatr. Surg., 19: 33-40, 1984.

17) Tibboel, D., A.W.M. van der Kamp and J.C. Molenaar: The effect of experimentally induced intestinal perforation at an early developmental stage. J. Pediatr. Surg., 16: 1017-1020, 1981.

18) Tibboel, D., A.W.M. van der Kamp and J.C. Molenaar: An experimental study of the effect of an intestinal perforation at various developmental stages. Z. Kinderchir, 37: 62-66, 1982.

19) Tibboel, D., P. Klück, A.W.M. van der Kamp, Ch. Vermeykeers and J.C. Molenaar: The development of the characteristic anomalies found in gastroschisis: Experimental and clinical data. Z. Kinderchir, 40: 355-360, 1985.

20) Tibboel, D., P. Raine, M. McNee, A. Azmy, P. Klück, D. Koung and J.C. Molenaar: Developmental aspects of gastroschisis. J. Pediatr. Surg., 21: 865-869, 1986.

21) Wesson, D.E., T. Muraji, G. Kent, R.M. Filler and T. Almalchi: The effect of intrauterine esophageal ligation on growth of fetal rabbits. J. Pediatr. Surg., 19: 398-399, 1984.

\section{Explanation of Figures}

\section{Plate I}

The schema on the right of each photograph shows the change in positions of the proximal and distal limbs during rotation of the midgut loop.

abbreviations (Figs. 2-6): p.; proximal limb of the midgut loop, d.; distal limb of the midgut loop, Om.; omphalo-mesenteric artery, arrow; apex of the loop.

Fig. 2. Stage 27 (5 days of incubation).

Shows the L-shaped midgut, or the midgut loop. The omphalo-mesenteric artery runs toward the apex of the loop. $\times 11$.

Fig. 3. Stage 28 (5.5 days of incubation).

Beginning of rotation of the loop. Only the proximal limb slightly rotates counterclockwise around the axis of the omphalomesenteric artery. $\times 9$.

Fig. 4. Stage 31 ( 7 days of incubation).

Both the proximal and distal limbs rotate counterclockwise through $90^{\circ}$ around the artery. $\times 10$ 

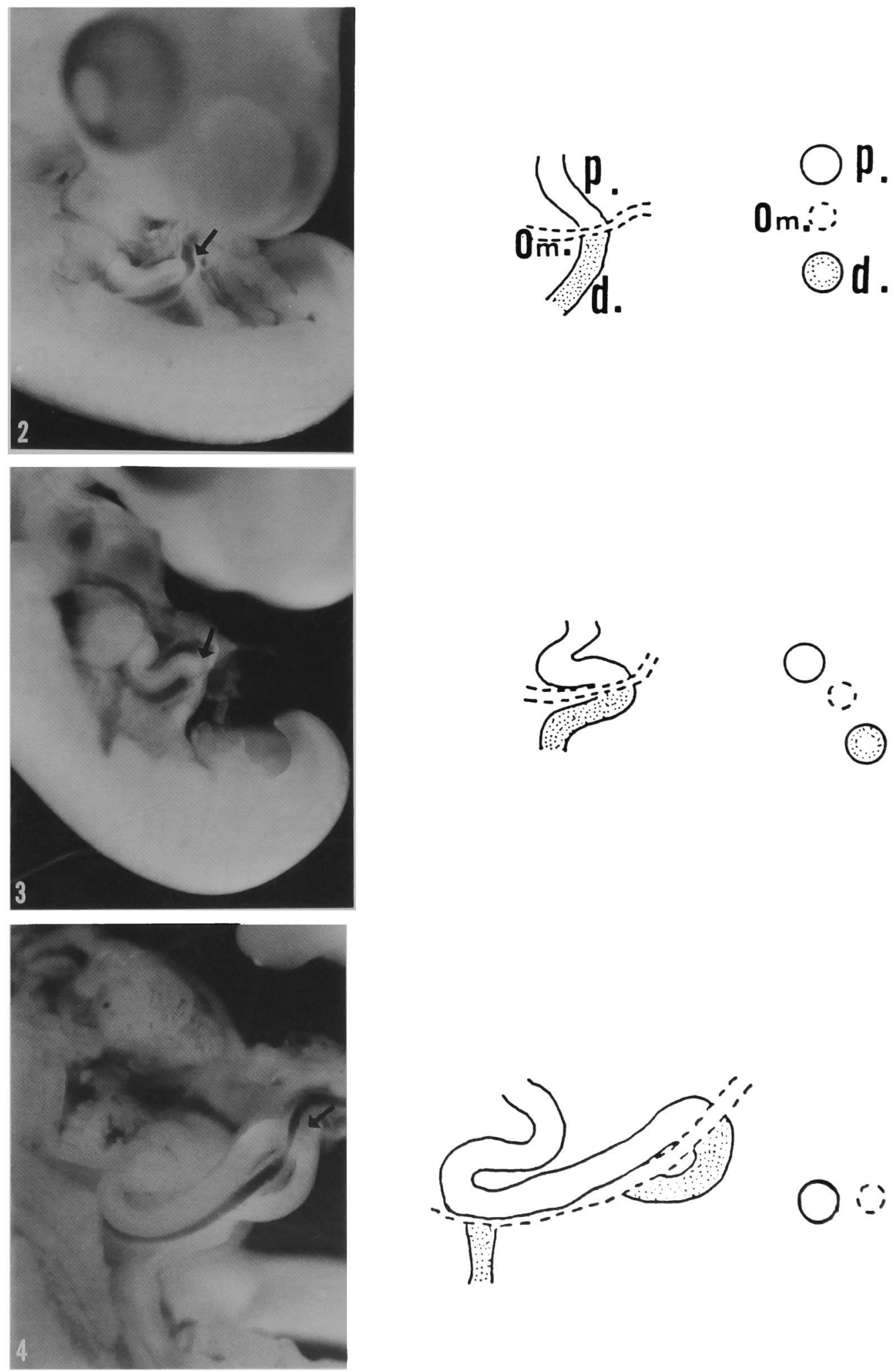

$0: 0$ 
Plate II
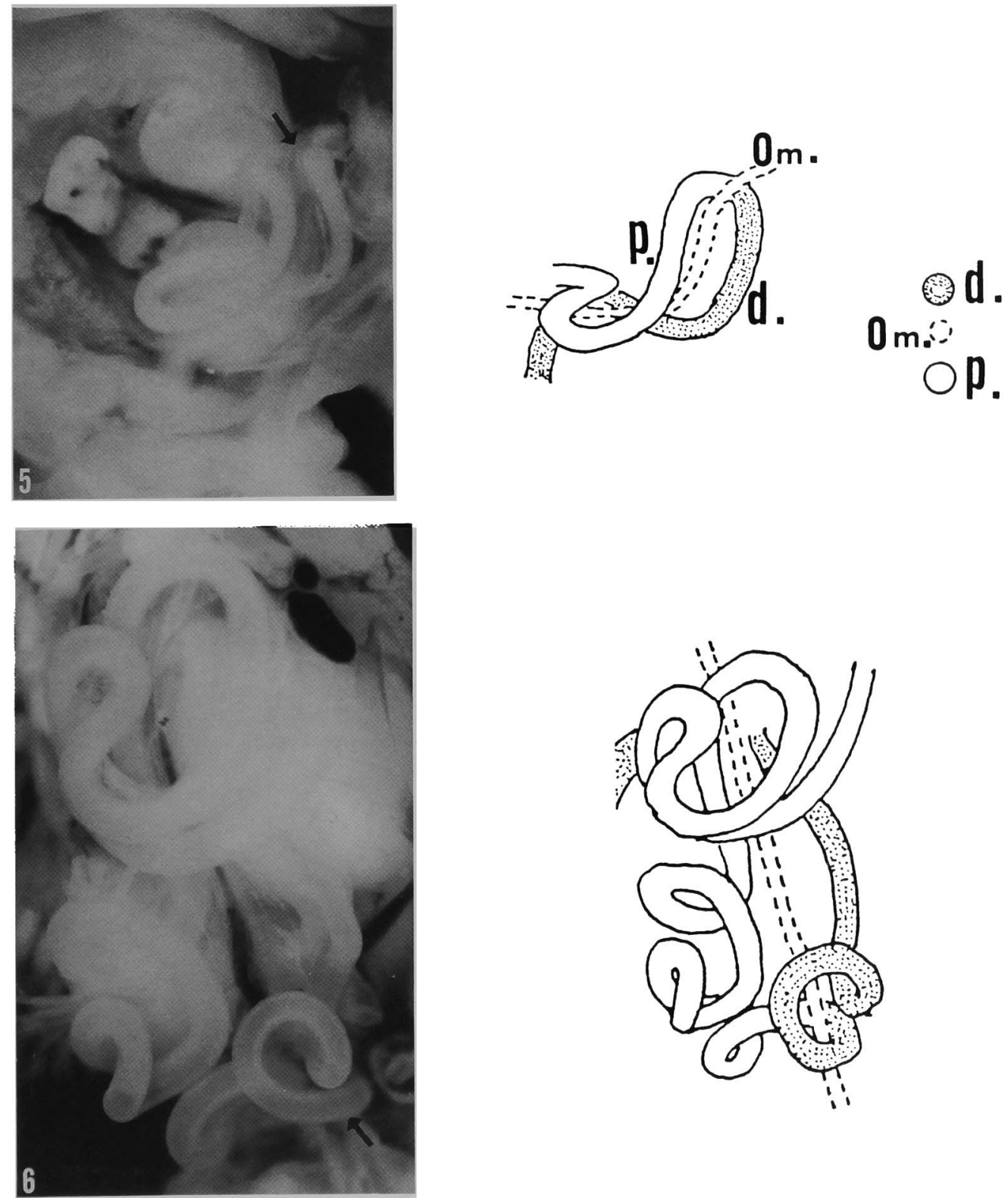

Plate II

Fig. 5. Stage 36 (10 days of incubation).

Both the proximal and distal limbs rotate counterclockwise through $180^{\circ}$ around the artery and the proximal limb shifts below the distal one. $\times 9$.

Fig. 6. Stage 40 (14 days of incubation).

The intestine is elongated and forms secondary intestinal loops by convoluting 5-6 times. $\times 6$. 


\section{Plate III}

Transverse sections of developing intestine showing the process of structural completion (Figs. 7-11). Hematoxylin-Eosin staining.

Fig. 7. Stage 27.

Shows the wall of the midgut loop. The mucosa, submucosa and serosa are distinguished but the muscular layer is not observed. $\times 200$.

Fig. 8. Stage 31 .

Both the annular and longitudinal muscle fibers (arrow) appear, forming three layered structure (mucosa, muscle layers and serosa). $\times 180$.

Fig. 9. Stage 33 .

The luminal surface of mucosa is slightly undulated, roughly folded and the formation of villi is seen. $\times 200$.

Fig. 10. Stage 35 .

The villi of the mucosa, and the inner circular and outer longitudinal muscle layers have well developed. $\times 150$.

Fig. 11. Stage 39.

Well-developed villi are observed, and the muscle layers have become more thickened. $\times 150$.

Fig. 12. Dilated portion of the intestine (see the text). Usual three-layered structure (mucosa, muscle layers and serosa) can be observed, but the intestinal wall (arrow), as a whole, is thinned. below; undilated distal stump. $\times 75$.

\section{Plate IV}

Fig. 13. Shows extreme dilatation of the proximal potion to the transected site. In the cases, transected ends, the stumps are closed by membranous obliteration. This dilatated intestine developed without convolution. Retention of meconium (green colored) in the lumen of intestine is seen (Figs. 12-14).

arrow indicates the proximal stump: common to Figs. $12-14 . \times 13$.

Fig. 14. Another case of dilatation of the intestine. Two stumps are closed by tissue repair (see the text) and apart from each other. $\times 10$.

Fig. 15 In this case dilatation of the intestine proximal to the transected site was absent and the secondary convolution of the portion occurred as in the normal embryo. $\times 8$. 
Plate III
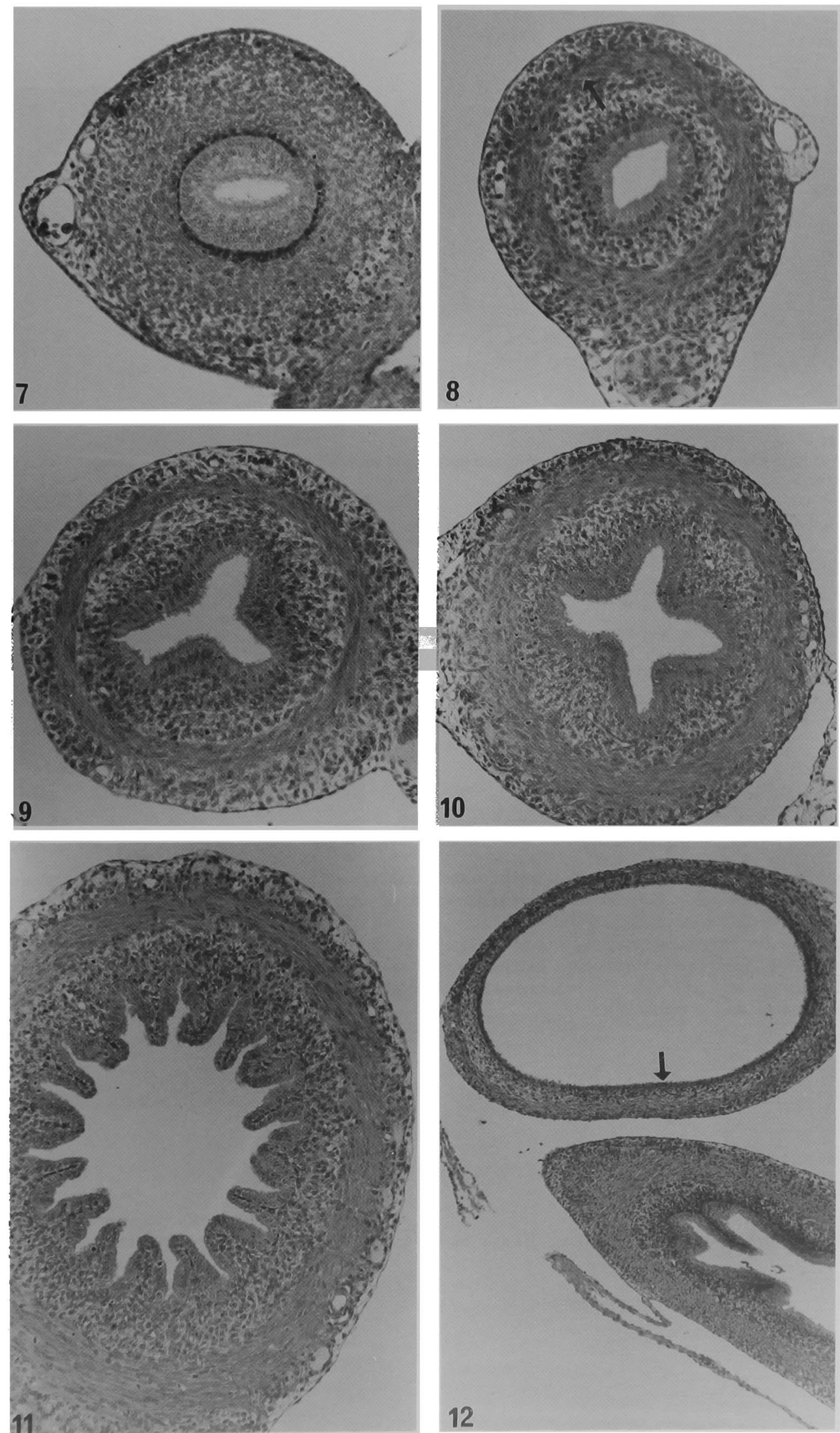
Plate II
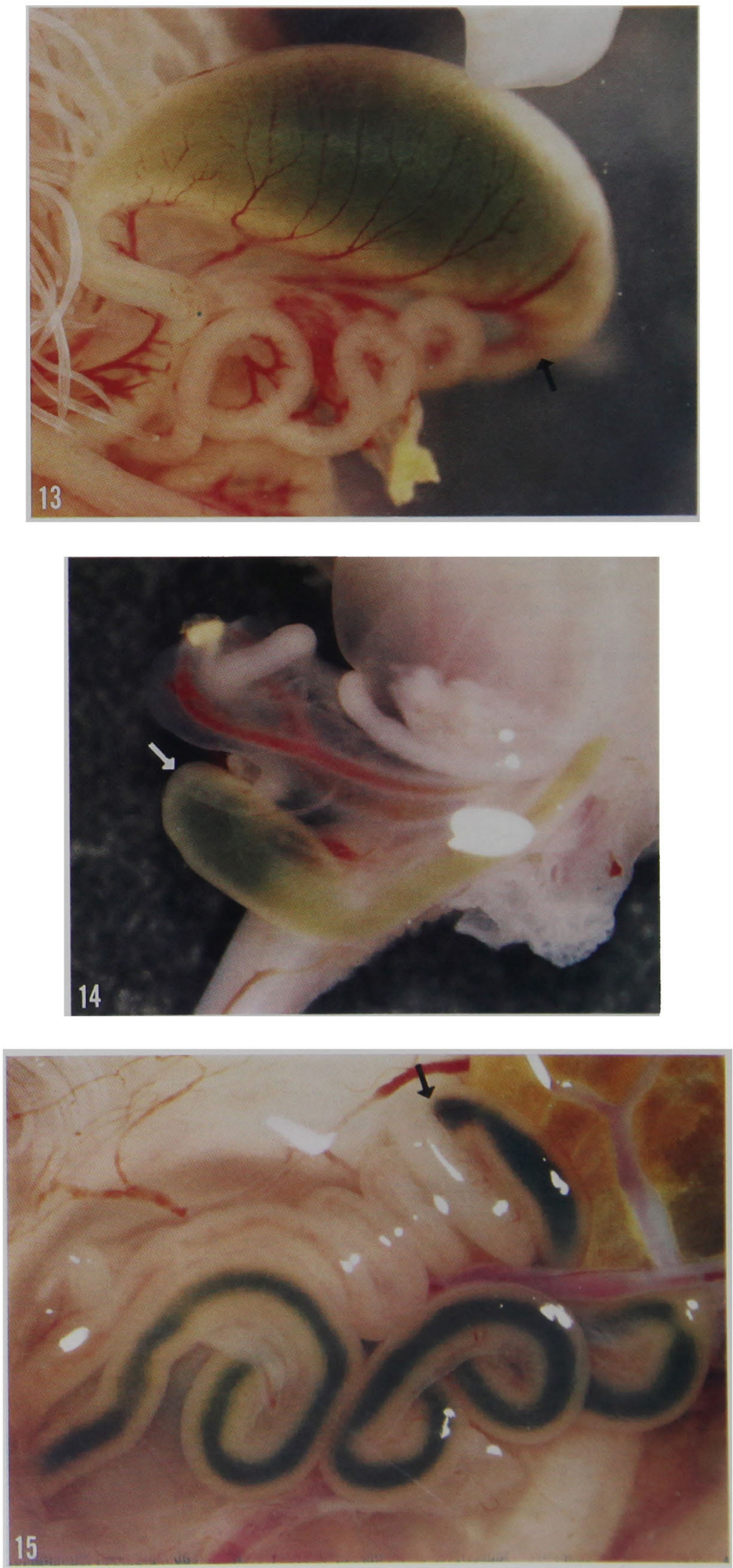